\title{
On a Fixed Point Theorem by Brosowski and Singh
}

\author{
A. Carbone
}

\begin{abstract}
We generalize a fixed point theorem of B. Brosowski and S. P. Singh to multi-valued non-expansive maps in Banach spaces with convex structure. Such maps have applications, for example, in game theory and in the mathematical modelling of some economical problems.
\end{abstract}

Keywords: Fixed point theorems, multi-valued maps, non-expansive maps, contractive maps, best approximations, convex structures

AMS subject classification: 47 H 10, 47 H 04, 47 H 09, 41 A 65, 46 A 99

Let $C$ be a non-empty set in a real normed space $X$. A convex structure on $C$ is, by definition, a continuous map $F:[0,1] \times C \times C \rightarrow C$ having the two properties

$$
\begin{aligned}
F(\lambda, x, F(\mu, y, z)) & =F\left(\lambda+(1-\lambda) \mu, F\left(\frac{\lambda}{\lambda+(1-\lambda) \mu}, x, y\right), z\right) \\
F(\lambda, x, x) & =x
\end{aligned}
$$

for $\lambda, \mu \in[0,1]$ with $\lambda+(1-\lambda) \mu \neq 0$ and $x, y, z \in C$. From (1) and (2) it follows that

$$
\left.\begin{array}{l}
F(1, x, y)=x \\
F(0, x, y)=y
\end{array}\right\} \quad(x, y \in C) \text {. }
$$

The general theory of convex structures has applications, for example, in game theory and mathematical economics [10], but also in some problems of colour vision and petroleum engineering $[4,7,8]$. A nice survey on basic properties and applications of convex structures may be found in [5].

Obviously, every convex set $C$ in a normed space $X$ gives rise to a canonical convex structure by putting $F(\lambda, x, y)=\lambda x+(1-\lambda) y$. Another example may be obtained as follows: Given a Hilbert space $H$, let $C$ be the set of all self-adjoint positive definite operators in $X=\mathcal{L}(H)$. For $\lambda \in[0,1]$ and $A, B \in C$ we put $F(\lambda, A, B)=A^{\lambda} B^{1-\lambda}$, where $A^{\lambda}$ denotes the usual fractional power (see, e.g., [3])

$$
A^{\lambda} x=\frac{\sin \pi \lambda}{\pi} \int_{0}^{\infty} \tau^{\lambda-1}(A-\tau I)^{-1} x d \tau \quad(0<\lambda<1) .
$$

A. Carbone: Università della Calabria, Dipartimento di Matematica, I - 87036 Arcavacata di Rende (CS), Italy 
In what follows, we assume throughout that the convex structure $F$ is regular in the sense that

$$
\|F(\lambda, x, p)-F(\lambda, y, q)\| \leq \varphi(\lambda)\|x-y\|+(1-\psi(\lambda))\|p-q\|
$$

for $\lambda \in[0,1]$ and $x, y, p, q \in C$, where $\varphi, \psi:[0,1] \rightarrow[0,1]$ are continuous functions satisfying

$$
\varphi(0)=\psi(0)=0, \quad \varphi(1)=\psi(1)=1, \quad 0<\varphi(\lambda), \psi(\lambda)<1 \text { for } 0<\lambda<1 .
$$

Of course, the canonical convex structure on a convex set is always regular with $\varphi(\lambda)=$ $\psi(\lambda)=\lambda$. Finally, we recall that a set $D \subseteq C$ is called $F$-starshaped if there exists a point $p \in D$ such that $F(\lambda, x, p) \in D$ for all $\lambda \in[0,1]$ and $x \in D$.

Theorem. Given a real normed space $X$ and a non-empty closed set $C \subset X$ with regular convex structure $F:[0,1] \times C \times C \rightarrow C$, let $f: C \rightarrow 2^{X}$ be a multi-valued map with compact values, $f(\partial C) \subseteq C$ and some fixed point $x_{0} \in C$, i.e. $x_{0} \in f\left(x_{0}\right)$. Suppose the following:

(i) The set $D$ of best $C$-approximants to $x_{0}$, i.e.

$$
D=\left\{x: x \in C \text { such that }\left\|x_{0}-x\right\|=d\left(x_{0}, C\right)\right\}
$$

is non-empty and F-starshaped.

(ii) The map $f$ is non-expansive on $D$ with respect to the Hausdorff metric $h$ on $X$,

$$
h\left(f(x), f\left(x_{0}\right)\right) \leq\left\|x-x_{0}\right\| \quad(x \in D),
$$

and $f(D)$ is compact.

Then $f$ has a fixed point closest to $x_{0}$.

Proof. Observe first that all points $x \in D$ must belong to the boundary $\partial C$ of $C$. In fact, if $x \in D$ were an interior point of $C$, we would have $x_{\lambda}=F\left(\lambda, x_{0}, x\right) \in C$ and

$$
\left\|x_{\lambda}-x_{0}\right\|=\left\|F\left(\lambda, x_{0}, x\right)-F\left(\lambda, x_{0}, x_{0}\right)\right\| \leq(1-\psi(\lambda))\left\|x-x_{0}\right\|<\left\|x-x_{0}\right\|
$$

for $\lambda>0$ sufficiently small, contradicting the fact that $x$ is a point of best approximation to $x_{0}$ in $C$.

The inclusion $D \subset \partial C$ implies that $f$ maps the set $D$ into itself: for $x \in D$ and $y \in f(x)$ we have $y \in f(\partial C) \subseteq C$ and

$$
\left\|x_{0}-y\right\| \leq h\left(f\left(x_{0}\right), f(x)\right) \leq\left\|x_{0}-x\right\|=d\left(x_{0}, C\right)
$$

by (6), hence $y \in D$.

Since $D$ is $F$-starshaped, we may choose $p \in D$ such that $F(\lambda, x, p) \in D$ for all $\lambda \in[0,1]$ and $x \in D$. Now let $\left(\lambda_{n}\right)_{n}$ be a sequence in $(0,1)$ converging to 1 , as $n \rightarrow \infty$, and define $f_{n}: D \rightarrow 2^{X}$ by

$$
f_{n}(x)=\bigcup_{y \in f(x)} F\left(\lambda_{n}, y, p\right) \quad(x \in D) .
$$


Since $D$ is $F$-starshaped, $f_{n}(x)$ is actually a subset of $D$ for any $x \in D$. We claim that $f_{n}$ is a multi-valued contraction on $D$. In fact, fix $x_{i} \in D$ and choose $z_{i} \in f_{n}\left(x_{i}\right)$, hence $z_{i}=F\left(\lambda_{n}, y_{i}, p\right)$ for some $y_{i} \in f\left(x_{i}\right) \quad(i=1,2)$. We get then the estimate

$$
\begin{aligned}
\left\|z_{1}-z_{2}\right\| & =\left\|F\left(\lambda_{n}, y_{1}, p\right)-F\left(\lambda_{n}, y_{2}, p\right)\right\| \\
& \leq \varphi\left(\lambda_{n}\right)\left\|y_{1}-y_{2}\right\| \leq \varphi\left(\lambda_{n}\right) h\left(f\left(x_{1}\right), f\left(x_{2}\right)\right) \leq \varphi\left(\lambda_{n}\right)\left\|x_{1}-x_{2}\right\|,
\end{aligned}
$$

since $F$ is regular and $f$ is non-expansive on $D$. We conclude that the map $f_{n}$ is a multi-valued contraction, and thus, by Nadler's fixed point theorem [6], there exists $x_{n} \in D$ such that $x_{n} \in f\left(x_{n}\right)$.

Now we employ the hypothesis that $f(D)$ is compact and choose a subsequence $\left(x_{n_{k}}\right)_{k}$ such that $x_{n_{k}} \rightarrow x_{*}$ as $k \rightarrow \infty$. The relation $x_{n_{k}} \in f_{n_{k}}\left(x_{n_{k}}\right)$ means that $x_{n_{k}}=F\left(\lambda_{n_{k}}, y_{n_{k}}, p\right)$ for some $y_{n_{k}} \in f\left(x_{n_{k}}\right)$, hence $y_{n_{k}} \rightarrow y_{*}$ for some $y_{*} \in f\left(x_{*}\right)$. By the continuity of $F$, this implies that

$$
x_{n_{k}}=F\left(\lambda_{n_{k}}, y_{n_{k}}, p\right) \longrightarrow F\left(1, y_{*}, p\right)=y_{*} \quad(k \rightarrow \infty)
$$

From (8) we conclude that

$$
d\left(x_{*}, f\left(x_{*}\right)\right) \leq\left\|x_{*}-x_{n_{k}}\right\|+\left\|x_{n_{k}}-y_{*}\right\|+d\left(y_{*}, f\left(x_{*}\right)\right) \rightarrow 0 \quad(k \rightarrow \infty)
$$

which shows that $x *$ is a fixed point of $f$ in $D$, since $D$ is closed

Our theorem may be considered as a generalization of a fixed point theorem of $S$. $P$. Singh [9] (where $f$ is single-valued and $C$ is star-shaped in the usual sense) which in turn extends a fixed point theorem of B. Brosowski [1] (where $f$ is linear and $C$ is convex). Moreover, some results of our recent paper [2] may be regarded as special variants of the above theorem for single-valued non-expansive maps $f$.

\section{References}

[1] Brosowski, B.: Fixpunktsätze in der Approximationstheorie. Mathematica (Cluj) 11 (1969), $195-220$.

[2] Carbone, A.: Some results on invariant approximation. Int. J. Math. Sci. 17 (1994), 483 - 487.

[3] Dunford, N. and J. T. Schwartz: Linear Operators. Part II: Spectral Theory. Self Adjoint Operators in Hilbert Spaces. Leyden: Int. Publ. 1963.

[4] Gudder, S. P.: Convexity and mixiures. SIAM Rev. 19 (1977), 221 - 240.

[5] Gudder, S. P.: A general theory of convexity. Rend. Sem. Mat. Milano 49 (1979), 89 96.

[6] Nadler, S. B.: Multivalued contraction mappings. Pacific J. Math. 30 (1969), 475 - 488.

[7] Rusin, M.: A new method for representing nonlinear blending problems in a linear programming format. SIAM J. A ppl. Math. 20 (1971), 143 - 164.

[8] Rusin, M.: The structure of nonlinear blending models. Chem. Engin. Sci. 30 (1975), $937-944$. 
[9] Singh, S. P.: An application of a fixed point theorem to approximation theory. J. Approx. Theory 25 (1979), 89 - 80.

[10] von Neumann, J: and O. Morgenstern: Theory of Games and Economics. Princeton: Princeton Univ. Press 1944.

Received 10.04.1995 